Article

\title{
Reversibility of the Magnetocaloric Effect in the Bean-Rodbell Model
}

\author{
Luis M. Moreno-Ramírez *(D) and Victorino Franco *(D) \\ Department of Condensed Matter Physics, ICMS-CSIC, Universidad de Sevilla, P.O. Box 1065, 41080 Sevilla, Spain \\ * Correspondence: lmoreno6@us.es (L.M.M.-R.); vfranco@us.es (V.F.)
}

check for updates

Citation: Moreno-Ramírez, L.M.;

Franco, V. Reversibility of the

Magnetocaloric Effect in the

Bean-Rodbell Model.

Magnetochemistry 2021, 7, 60.

https://doi.org/10.3390/

magnetochemistry7050060

Academic Editor: Joan-Josep Suñol

Received: 19 April 2021

Accepted: 30 April 2021

Published: 5 May 2021

Publisher's Note: MDPI stays neutral with regard to jurisdictional claims in published maps and institutional affiliations.

\begin{abstract}
The applicability of magnetocaloric materials is limited by irreversibility. In this work, we evaluate the reversible magnetocaloric response associated with magnetoelastic transitions in the framework of the Bean-Rodbell model. This model allows the description of both second- and first-order magnetoelastic transitions by the modification of the $\eta$ parameter $(\eta<1$ for second-order and $\eta>1$ for first-order ones). The response is quantified via the Temperature-averaged Entropy Change (TEC), which has been shown to be an easy and effective figure of merit for magnetocaloric materials. A strong magnetic field dependence of TEC is found for first-order transitions, having a significant increase when the magnetic field is large enough to overcome the thermal hysteresis of the material observed at zero field. This field value, as well as the magnetic field evolution of the transition temperature, strongly depend on the atomic magnetic moment of the material. For a moderate magnetic field change of $2 \mathrm{~T}$, first-order transitions with $\eta \approx 1.3-1.8$ have better TEC than those corresponding to stronger first-order transitions and even second-order ones.
\end{abstract}

Keywords: magnetocaloric effect; reversible response; Bean-Rodbell model

\section{Introduction}

Magnetocaloric (MC) materials deserve the attention of the research community due to their possible application in solid-state refrigeration at room temperature [1-3]. Prototypes of this technology have been shown to be energy efficient and environmentally friendly, aspects that are highly desired from the point of view of sustainability $[4,5]$. The MC effect [6] is defined as the temperature change (or entropy change) produced by the application/removal of a magnetic field in adiabatic (or isothermal) conditions, $\Delta T_{\text {ad }}$ (or $\Delta S_{i s o}$ ). MC materials are classified according to the order of the thermomagnetic phase transition $[7,8]$, being of first- or second-order type (FOPT and SOPT MC materials, respectively). Typically, SOPT materials present a moderate response in a wide temperature range, while FOPT materials present higher effects but in a narrow temperature span [9]. Besides this, another important characteristic of MC materials is the reversibility of their response, being fully reversible in the case of SOPT materials and not completely reversible for FOPT ones due to the associated hysteresis $[8,10]$. For practical applications in magnetic refrigeration, due to the cyclic operation of the devices, a large reversible response is a fundamental requirement. Therefore, the analysis of the reversible response is crucial to evaluate the actual MC performance of a material.

The MC performance of the materials in refrigeration devices has been extensively studied [8,11-17]. The most used criterion is the Relative Cooling Power $(R C P)$, which accounts for the heat that can be exchanged between the hot and cold reservoirs [18]. The $R C P$ is defined as:

$$
R C P=\Delta S_{i s o}^{\max } \Delta T_{F W H M},
$$

where $\Delta S_{i s o}^{\max }$ is the maximum value of $\Delta S_{i s o}$ at a certain magnetic field change and $\Delta T_{F W H M}$ is the Full Width at Half Maximum (FWHM) of $\Delta S_{i s o}(T)$. Equation (1) assumes that the temperature of the hot and cold reservoirs corresponds to $\Delta T_{F W H M}$. This approximation 
leads to erroneous interpretation of the potential applicability of materials. Materials with low $\Delta S_{i s o}$ can have $\Delta T_{F W H M}$ of hundreds of kelvins which, in fact, are impossible to achieve in real devices as $\Delta T_{a d}$ values are far from those ranges [19-21]. This misinterpretation can lead to argue the goodness of SOPT materials with very low MC responses in comparison to Gd or well-known FOPT MC materials.

To overcome this main issue, L. D. Griffith et al. proposed the use of the Temperature averaged Entropy Change (TEC) to evaluate the MC materials [22]. It is defined as:

$$
\operatorname{TEC}\left(\Delta T_{\text {lift }}\right)=\frac{1}{\Delta T_{\text {lift }} \max _{\text {mid }}}\left(\int_{T_{\text {mid }}-\frac{\Delta T_{\text {lift }}}{2}}^{T_{\text {mid }}+\frac{\Delta T_{\text {lift }}}{2}} \Delta S_{\text {iso }} \mathrm{d} T\right)
$$

where $\Delta T_{\text {lift }}$ is the working range of temperatures (differences between cold and hot reservoirs) and $T_{\text {mid }}$ is the middle temperature of the range. To obtain reliable values and to use this magnitude as a figure of merit for the MC materials, realistic values of $\Delta T_{\text {lift }}$ have to be employed. The authors proposed two strategies: 1) $\Delta T_{\text {lift }}$ of $3 \mathrm{~K}$ characteristic of a single layer in a device [23,24]; and 2) $\Delta T_{\text {lift }}$ of $10 \mathrm{~K}$ covering best $\Delta T_{a d}$ known today $(\approx 9 \mathrm{~K})$ under magnetic fields produced by permanent magnets $(\approx 2 \mathrm{~T})$ [25]. Using these criteria, the goodness of FOPT MC materials with respect SOPT ones was shown, in agreement with experimental observations [22].

In this work, we evaluate the reversible entropy change of materials undergoing magnetoelastic transitions in the framework of the Bean-Rodbell model [26] using TEC as a figure of merit. This model makes it possible to reproduce SOPT and FOPT materials by modifying the $\eta$ parameter, showing a good agreement with experimental results of MC materials [27-30]. In the present work, we show that the reversible TEC has a strong magnetic field dependence for FOPT, exhibiting a significant increase when the magnetic field is large enough to overcome the thermal hysteresis of the material observed at zero field. Besides the influence of $\eta$, which significantly increases the thermal hysteresis of the system, the atomic magnetic moment has been found to play a significant role on the reversible magnetocaloric performance through the field dependence of the transition temperature. In addition, for conventional magnetic field changes of $2 \mathrm{~T}$, the reversible TEC as a function $\eta$ and magnetic moment is found to have a maximum lying in the FOPT range, showing that FOPT with moderate hysteresis have larger MC performance than stronger FOPT, SOPT or even materials at the critical point between first- and second-order phase transition.

\section{Methods}

The Bean-Rodbell model [26] was originally developed to reproduce the magnetoelastic transition in MnAs alloy and it is based on the assumption that the relative volume change $(w)$ affects the transition temperature $\left(T_{t}\right)$ according to:

$$
T_{t}=T_{0}(1+\beta w)
$$

where $T_{0}$ is the transition temperature in absence of volume changes and $\beta$ an introduced parameter. According to this model, the magnetization $(M)$ is expressed as:

$$
\frac{M}{M_{S}}=B_{J}\left(\frac{g \mu_{B} J}{k_{B} T} \mu_{0} H+\frac{3 T_{0}}{T}\left(\frac{J}{J+1}\right) \frac{M}{M_{S}}+\eta \frac{9}{10}\left(\frac{2 J^{2}+2 J+1}{(J+1)^{3}}\right) \frac{T_{0}}{T} \frac{M^{3}}{M_{S}{ }^{3}}\right)
$$

where $M_{S}$ is the saturation magnetization $\left(M_{S}=n m\right.$, being $n$ the atomic magnetic moment density and $m$ the atomic magnetic moment, $\left.m=g J \mu_{B}\right), g$ the Landé factor, $\mu_{B}$ the Bohr 
magneton, $J$ the total quantum angular momentum, $B_{J}$ the Brillouin function, $\mu_{0}$ the permeability of vacuum, $H$ the magnetic field and $\eta$ a parameter of the model, defined as:

$$
\eta=\frac{5}{2}\left(\frac{(2 J+1)^{4}-1}{(2(J+1))^{4}}\right) \frac{M_{S}}{g \mu_{B} J} k_{B} T_{0} k \beta^{2},
$$

where $k$ is the compressibility modulus. The $\eta$ parameter controls the order of the transition, being of first-order type if $\eta>1$, second-order if $\eta<1$ and $\eta=1$ corresponds to the critical point between first- and second order phase transitions (where the order of the transition changes from second to first). In the framework of the Bean-Rodbell model, the quantification of the hysteresis and the reversible response is possible. This is determined from the single-valued solution of $T$ as function of $M$ and $H$ in Equation (4) [31]. For $\eta>1$, metastable and instable regions appear in the solution. The existence of the instable region leads to two different solutions when the material is heated up or cooled down, originating the associated hysteresis. In this work, fixed values for $g, T_{0}, n$ and $k$ were used $(2,300 \mathrm{~K}$, $3.0 \times 10^{28} \mathrm{~m}^{-3}$ and $2.5 \times 10^{-11} \mathrm{~Pa}^{-1}$, respectively), while $m$ (or $J$ ) and $\eta$ were varied to study their influence. These fixed values are in the range of those for typical MC materials at room temperature (e.g., $n=3.1 \times 10^{28} \mathrm{~m}^{-3}$ and $k=2.6 \times 10^{-11} \mathrm{~Pa}^{-1}$ for Gd [32] and $n=1.6 \times 10^{28} \mathrm{~m}^{-3}$ and $k=0.9 \times 10^{-11} \mathrm{~Pa}^{-1}$ [29] for $\left.\mathrm{La}(\mathrm{Fe}, \mathrm{Si})_{13}\right)$.

To evaluate the $\mathrm{MC}$ response, $\Delta S_{\text {iso }}$ is calculated from magnetization data using Maxwell relations as:

$$
\Delta S_{i s o}(0 \rightarrow H)=\mu_{0} \int_{0}^{H}\left(\frac{\partial M}{\partial T}\right)_{H} d H^{\prime},
$$

where the different solution of Equation (4) can be introduced (i.e., the cooling and heating branches of the magnetization leads to a $\Delta S_{i s o}$ response associated with each branch). The reversible response is obtained as the intersection of the heating and cooling ones [33]. From that reversible $\Delta S_{i s o}$, the reversible TEC have been calculated according to Equation (2).

\section{Results and Discussion}

To illustrate the hysteretic behavior in terms of the Bean-Rodbell model, Figure 1 shows, as an example, the temperature dependence of $M$ and $\Delta S_{i s o}$ for an atomic magnetic moment of $7 \mu_{B}$ for two values of the $\eta$ parameter, which correspond to SOPT $(\eta=0.5)$ and FOPT cases $(\eta=1.5)$. It can be observed that for $\eta=0.5$ (Figure $1 \mathrm{a}, \mathrm{c}$ ) both cooling and heating branches of $M$ are the same as there is no hysteresis associated with the transition, leading to a $\Delta S_{\text {iso }}$ response that is fully reversible (shaded area), as expected for SOPT materials. In the case of $\eta=1.5, M$ is not reversible for zero field $(0 \mathrm{~T})$, while it becomes reversible for larger fields (2 T) (Figure 1b). For this case, the field in which the thermal hysteresis of the FOPT response vanishes is $1.35 \mathrm{~T}$ (denoted as critical field, $H_{C}$ ). This irreversibility of $M$ leads to different $\Delta S_{i s o}$ responses when heating or cooling. For the shown case and a field of $2 \mathrm{~T}$, the reversible response coincides with the one of the heating branch (Figure 1d). It is inferred, therefore, that hysteresis reduces the MC performance as the reversible response is smaller and narrower than the maximum achievable one for moderate magnetic fields (which corresponds to the cooling branch). However, it is clearly observed that the reversible response of the FOPT is larger when compared to the SOPT one. 

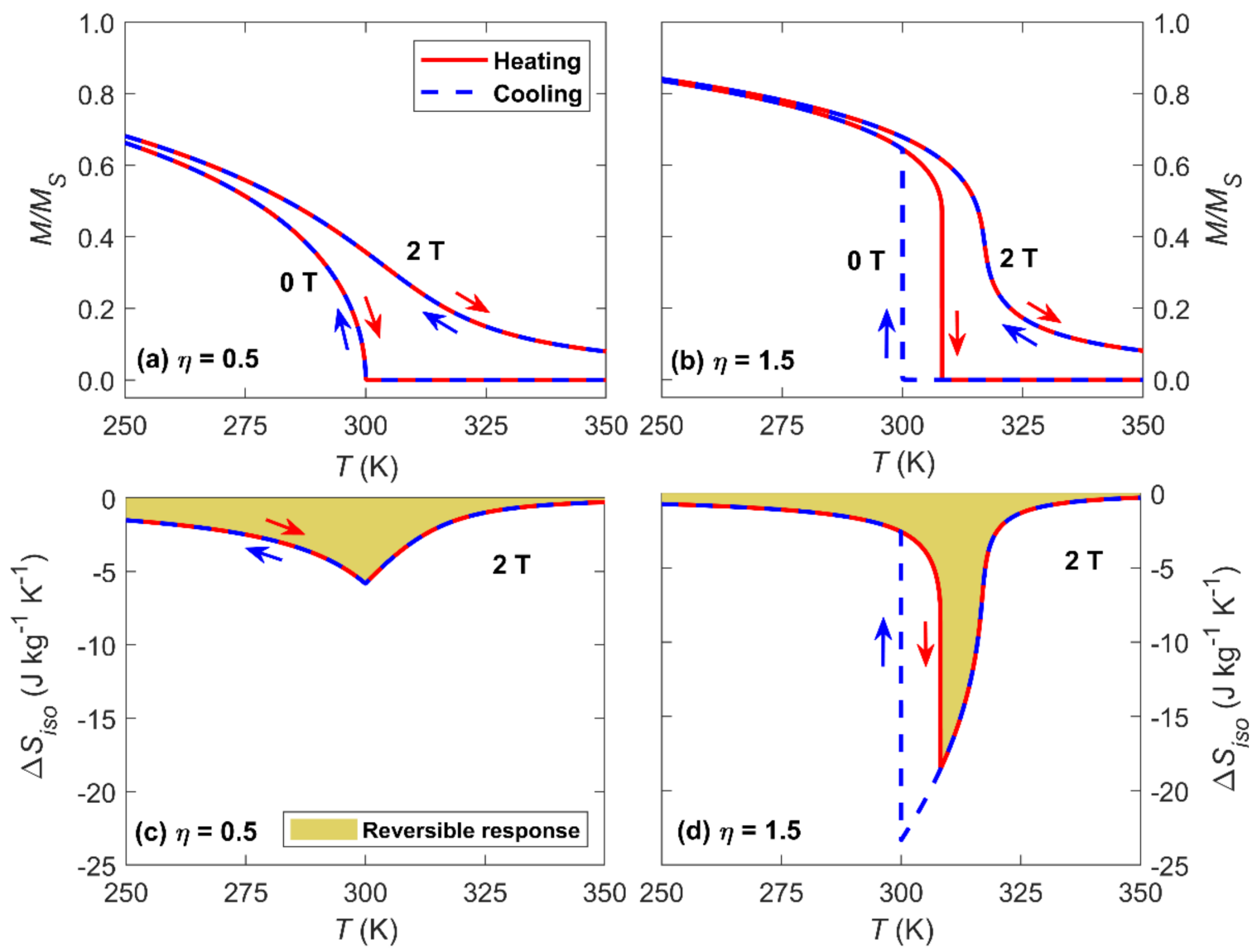

Figure 1. Temperature dependence of $M$ for an atomic magnetic moment of $7 \mu_{B}$ for 0 and $2 \mathrm{~T}$ for (a) $\eta=0.5$ (SOPT) and (b) $\eta=1.5$ (FOPT). Corresponding $\Delta S_{i s o}$ (from previous magnetization data) for (c) $\eta=0.5$ and (d) $\eta=1.5$.

With respect to field evolution, Figure 2a shows the magnetic field dependence of the maximum $\Delta S_{i s o}$ values for heating and cooling branches as well as the reversible contribution $\Delta S_{i s o}$ for $m=7 \mu_{B}$. For $\eta=1.5$ it is observed that both cooling and heating values start from significant values even for low fields (being larger for the former case as previously observed) in contrast to real cases. On the one hand, this can be ascribed to the modelling of the material as a single domain. However, this effect would only affect the magnetocaloric response at much lower fields than the ones of interest (a well-known $\mathrm{MC}$ material such as $\mathrm{La}(\mathrm{Fe}, \mathrm{Si})_{13}$ can be technically saturated around $0.25 \mathrm{~T}$ [34]). On the other hand, it can also be ascribed to the abrupt (instantaneous) transformation between FM to PM states for FOPT in the model (e.g., see Figure 1b), while coexistence among both phases during transformations is experimentally observed. This can be solved by including the kinetic process specific to each material [35], although this would require the inclusion of different additional models besides the Bean-Rodbell one (which is the main focus of this work). Continuing the discussion of Figure 2a, the reversible response remains much smaller than both heating and cooling cases up to a certain magnetic field (denoted by $H_{I}$ ) at which a significant increase of the response is observed. Above that magnetic field, the reversible response is the same as the heating one. This $H_{I}$ shows how important the magnetic field is in overcoming the limitations of the hysteresis (and improving the reversible response). It should be noted that the value of $H_{I}$ is smaller than $H_{C}(0.58 \mathrm{~T}$ vs. $1.35 \mathrm{~T}$ ). Analyzing the effect of the magnetic field on the transition temperature (inset of Figure 2a), it can be observed that $H_{I}$ corresponds to the magnetic field at which the transition temperature of the cooling branch reaches the value at zero field of the transition temperature of the heating branch, i.e.,:

$$
T_{t, \text { Heating }}(0)=T_{t, \text { Cooling }}\left(H_{I}\right) \text {. }
$$



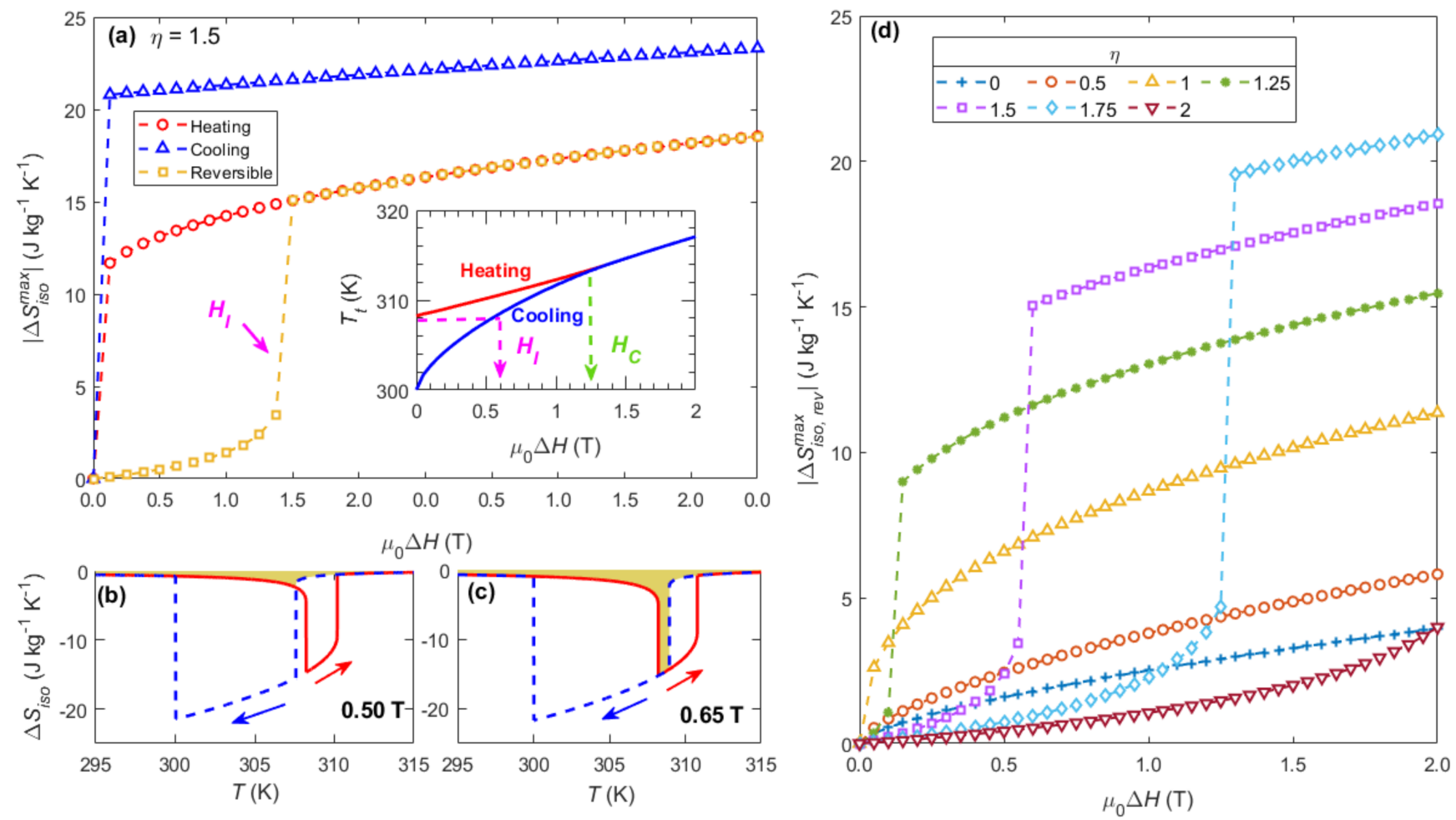

Figure 2. (a) Magnetic field dependence of the maximum value of $\Delta S_{i s o}$ and the evolution of the transition temperatures (inset) for $\eta=1.5$ and $m=7 \mu_{B}$; (b) Temperature dependence of $\Delta S_{i s o}$ for $\eta=1.5$ at $0.5 \mathrm{~T}$ and (c) $0.65 \mathrm{~T}$; (d) Magnetic field dependence of maximum reversible $\Delta S_{i s o}$ for different $\eta$ values and $m=7 \mu_{B}$.

This behavior can be understood as follows: (1) For magnetic fields below $H_{I}$, the temperatures of the peak of the cooling and heating responses are separated, and then the common response corresponds to the tail of the $\Delta S_{i s o}$ curves (Figure 2b). (2) For fields above $H_{I}$, the cooling response is broad enough to be at least partially overlapped with the heating peak and, due to the abrupt shape of the peak, the common response reaches the maximum of the heating peak (Figure 2c). It should be noted that as the transition temperatures of both branches are shifted to higher temperatures with increasing field, the maximum reversible response can only reach the one of the heating branch.

Now we extend the analysis to different values of the $\eta$ parameter. Figure $2 \mathrm{~d}$ shows the field dependence of the $\Delta S_{\text {iso,rev }}$ for different $\eta$ while keeping the magnetic moment to $7 \mu_{B}$. For all FOPT cases (i.e., $\eta>1$ ), the previous characteristic of an abrupt increase of the reversible $\Delta S_{i s o}$ at $H_{I}$ is observed (except for $\eta=2$ ). Moreover, it can be noted that $H_{I}$ increases as $\eta$ increases, i.e., as the FOPT character becomes more relevant (being for $\eta=2$ larger than the explored magnetic field range of $2 \mathrm{~T}$ ). For fields above $H_{I}$, as in the SOPT range, the maximum achievable value of $\Delta S_{i s o, r e v}$ increases with $\eta$. This plot reveals that $\Delta S_{i s o, r e v}$ of FOPT can be quite small if the magnetic field is not enough to overcome the hysteresis $\left(H<H_{I}\right)$ and those values are smaller than those of SOPT. However, if the field large enough to overcome the hysteresis, FOPT values are much larger than those of SOPT. This illustrates that at moderate magnetic field changes of 1-2 T, MC materials with moderate first-order character (i.e., significant heating/cooling magnetocaloric responses with small thermal hysteresis) have much better reversible performance than those with stronger first-order character (e.g., for $1 \mathrm{~T}$, it is preferable a FOPT with $\eta$ closer to 1.5 than to 2 ) or second-order ones. This is in agreement with experimental observations $[8,13,36]$. To further extend this discussion and to establish a reliable comparison between SOPT and FOPT materials, we can evaluate the reversible MC performance in the framework of TEC magnitude $\left(T E C_{r e v}\right)$. 
Figure 3a shows the magnetic field dependence of $T E C_{\text {rev }}$ for $\eta=1.5$ and $m=7 \mu_{B}$ using the two proposed $\Delta T_{\text {lift }}$ of 3 and $10 \mathrm{~K}$. It should be noted that the obtained values are similar to those of experimental measurements [22]. For both operating temperature spans, a significant increase of the values at $H_{I}$ can be observed, as could be expected from the significant increase of $\Delta S_{i s o, r e v}$. However, the increase in $T E C_{\text {rev }}$ is not as abrupt as the one previously observed for $\Delta S_{i s o, r e v}$. This is ascribed to the limited temperature width of the reversible response for fields close to $H_{I}$ in comparison to $\Delta T_{\text {lift }}$. Therefore, to obtain significant $T E C_{r e v}$ values we have to reach fields larger than $H_{I}$. These values of $T E C_{r e v}$ depend on the $\Delta T_{\text {lift }}$ employed, as can be observed from the figure. The dependence of $H_{I}$ on $\eta$ and $m$ (or $J$ ) parameters is shown in Figure 3b. A steep dependence with $\eta$ is clearly observed, showing that as the first-order character increases, higher magnetic field changes are needed to obtain a significant reversible response. Moreover, an interesting dependence with the atomic magnetic moment is also shown, being $H_{I}$ significantly reduced as $m$ increases. This leads to prefer large magnetic moments in order to have a better reversible performance at moderate magnetic field changes.
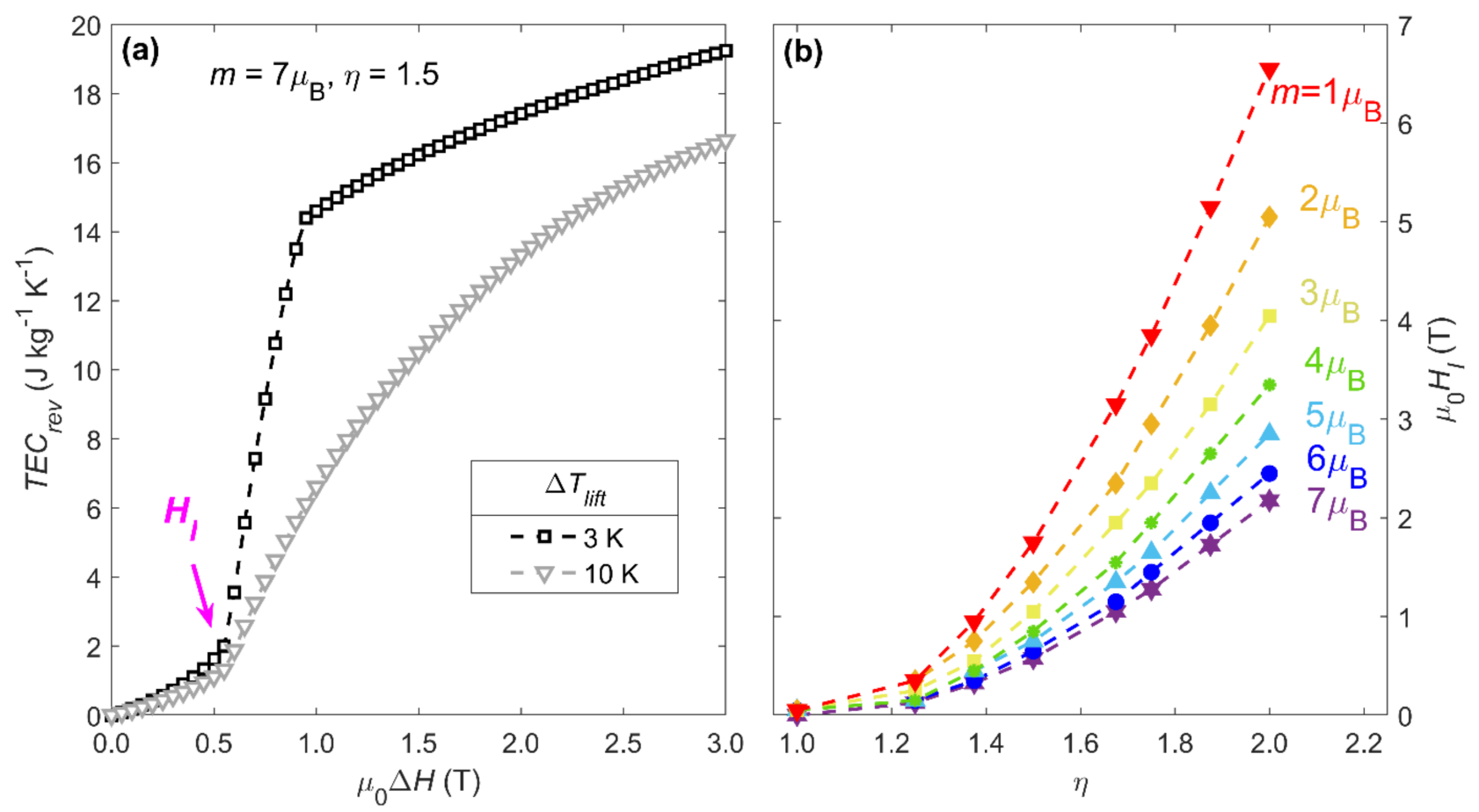

Figure 3. (a) Magnetic field dependence of the reversible TEC for $\eta=1.5$ and $m=7 \mu_{B}$ using a $\Delta T_{\text {lift }}$ of 3 and $10 \mathrm{~K}$; (b) $H_{I}$ as a function of $\eta$ for different atomic magnetic moments.

To further understand the influence of $m$ and $\eta$ parameters on reversibility, we analyze their influence on the thermal hysteresis at zero field and on the magnetic field dependence of the transition temperature (Figure $4 \mathrm{a}, \mathrm{b}$, respectively). A significant dependence on $\eta$ is observed for the thermal hysteresis, while $m$ has a much less relevant influence on $\Delta T_{\text {hys. }}$. This trend is similar to that previously observed for $H_{I}$ (Figure $3 \mathrm{~b}$ ), although the $m$ dependence is more relevant for $H_{I}$. The magnetic evolution of the transition temperature is significantly affected by $m$ (from 1 to $7 \mu_{B}$ the shift of transition temperature caused by field is doubled). This dependence of the transition temperature evolution is the main responsible of the $H_{I}$ dependence with $m$. With this, it can be concluded that, for large atomic magnetic moments, the larger $\frac{d T_{t}}{\mathrm{~d} H}$ values the better reversible TEC performance. 

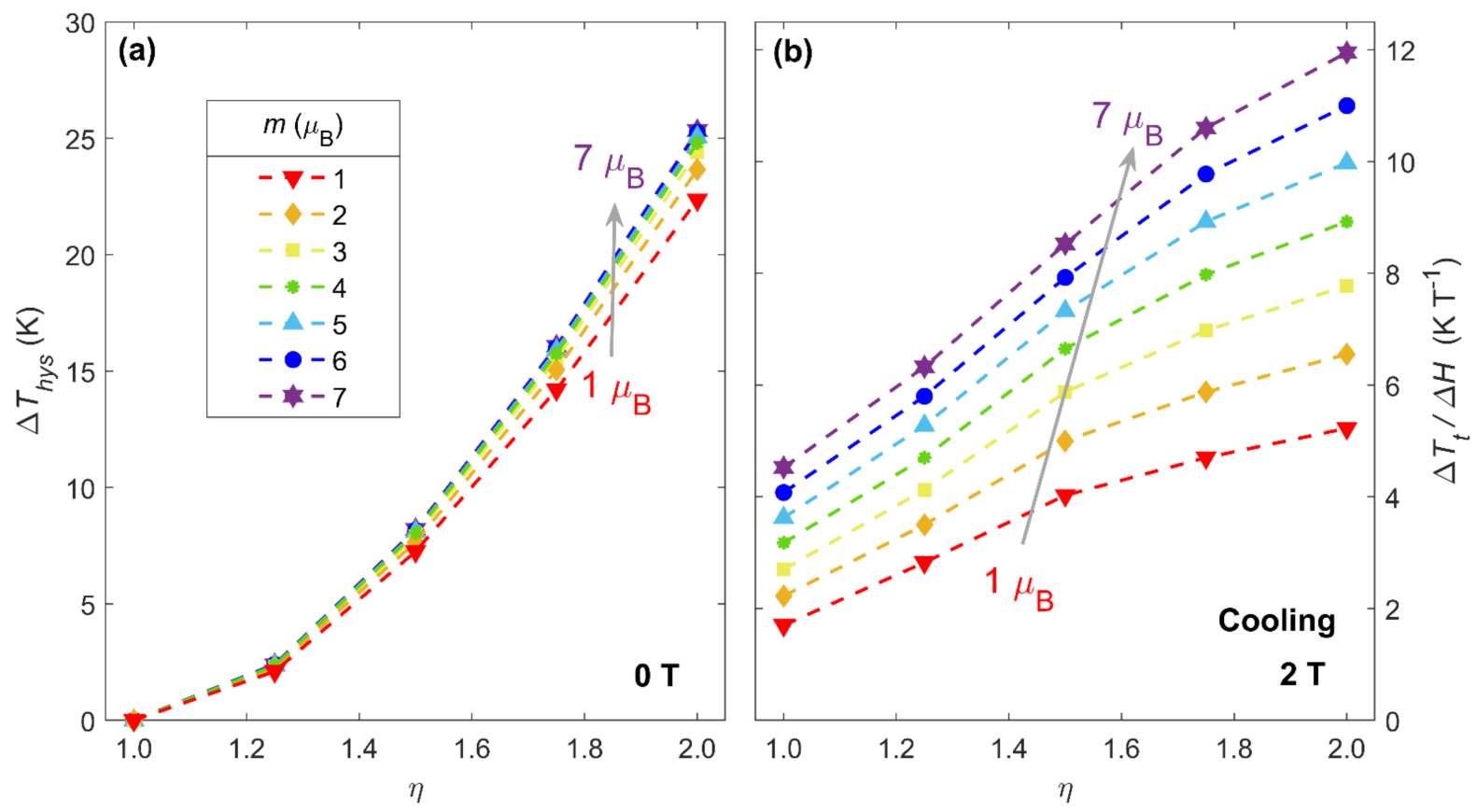

Figure 4. (a) Thermal hysteresis at zero field and (b) magnetic field evolution of the transition temperature (cooling branch) as a function of $\eta$ and $m$.

This increment of the sensitivity of the transition to the magnetic field can be understood in terms of the Clausius-Clapeyron formalism [37,38]. For an idealized FOPT, this leads to the equation:

$$
\frac{\mathrm{d} T_{t}}{\mathrm{~d} H}=-\frac{\Delta M}{\Delta S} .
$$

with $\Delta M$ and $\Delta S$ being the jump produced at the transition for the magnetization and entropy, respectively. Dissipative terms are not considered, as we are looking for an approximate value that represents the general trend, although they should be added for a detailed analysis $[39,40]$. For the atomic magnetic moment dependence, $\Delta M$ increases with increasing $m$ and, therefore, $\Delta S$ also increases. However, a larger increase of the magnetization jump when compared to the one of the entropy is observed which explains the increment of $\frac{\mathrm{d} T_{t}}{\mathrm{~d} H}$ with increasing $m$ by considering Equation (8). Following a similar behavior, $\Delta M$ and $\Delta S$ both increase with increasing $\eta$. However, the effect is more significant for $\Delta M$, leading to larger $\frac{\mathrm{d} T_{t}}{\mathrm{~d} H}$ with increasing $\eta$.

Finally, in Figure 5a we plotted the $\eta$ and $m$ dependence of $T E C_{\text {rev }}$ (using $\Delta T_{\text {lift }}$ of $3 \mathrm{~K}$ ) for a magnetic field change of $2 \mathrm{~T}$. With respect to the $\eta$ dependence, a nonmonotonic tendency is observed, reaching maximum values in the FOPT region (i.e., for $\eta>1$ ) independently of the value of the atomic moment. After that maximum, the values significantly decrease, becoming smaller than those of SOPT range. This decrease is ascribed to the associated thermal hysteresis, which cannot be overcome at the working magnetic field change of $2 \mathrm{~T}$. With respect to $m$ dependence, the maximum values of $T E C_{\text {rev }}$ $\left(T E C_{r e v}^{\max }\right)$ increase as $m$ increases. The reason for this is that, together with the increase of the atomic moment, the saturation magnetization value increases (in our model we do not modify the atomic density), promoting larger $\Delta S_{i s o}^{\max }$ values and therefore larger $T E C_{r e v}^{\max }$. Together with this, we observe that, as $m$ increases, the maximum values of $T E C_{r e v}^{\max }$ are shifted to higher $\eta$. These optimal values $\left(\eta^{o p t}\right)$ are plotted in Figure $5 \mathrm{~b}$ (left y-axis) as a function of $m$, showing a significant evolution. In addition, the values of $T E C_{r e v}^{\max }$ using $\Delta T_{\text {lift }}$ of 3 and $10 \mathrm{~K}$ are also included (right y-axis). The trend for $\eta^{o p t}$ can be understood as the shift of the transition temperature with the magnetic field significantly increases with $m$ (Figure $4 \mathrm{~b}$ ), making it possible to increase the thermal hysteresis that can be overcome by 
the field. It is found that the optimal performance in terms of the Bean-Rodbell model lies in the range of 1.3-1.8 for magnetic field changes of $2 \mathrm{~T}$ using 3 and $10 \mathrm{~K}$ as temperature span.

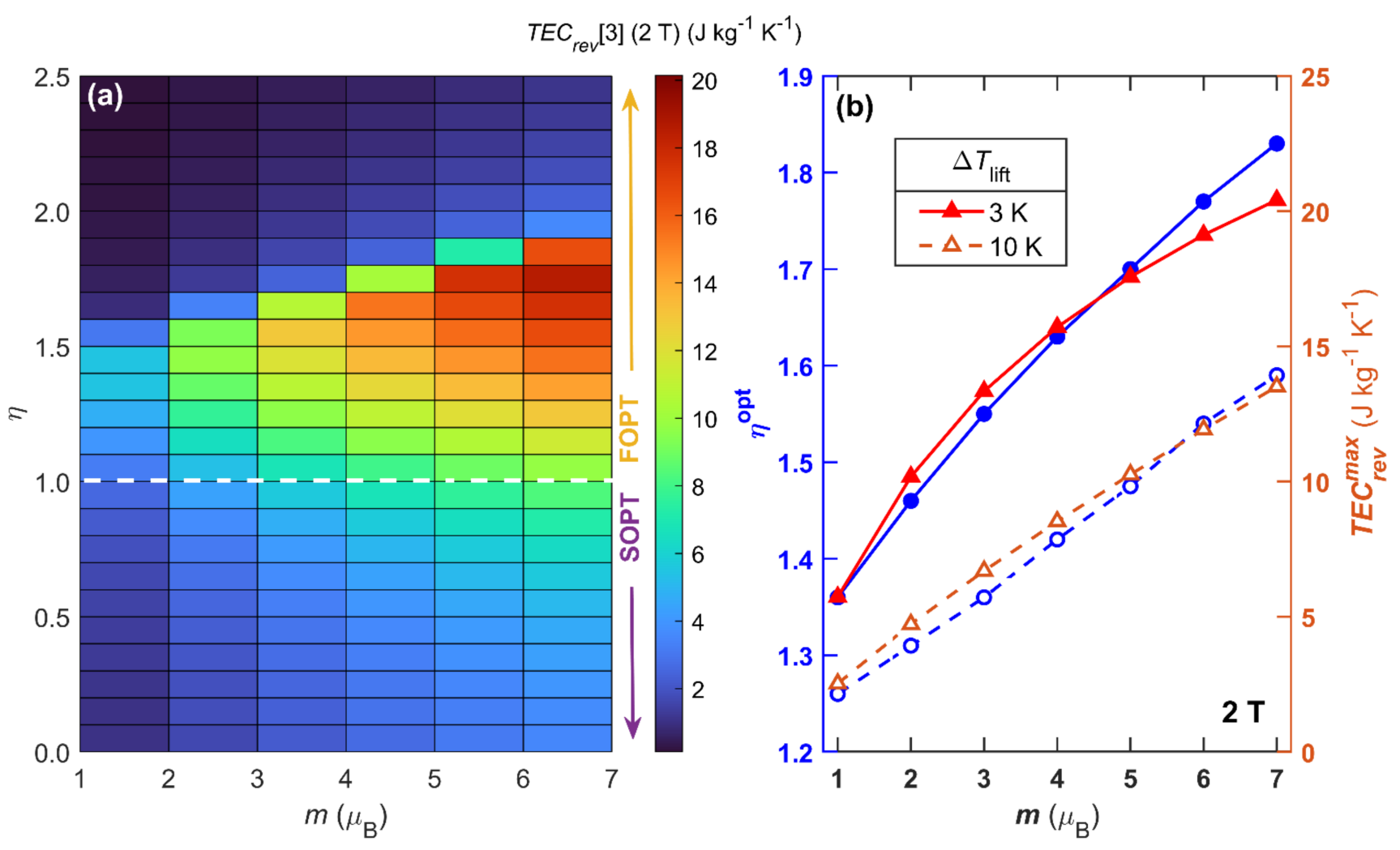

Figure 5. (a) $T E C_{\text {rev }}$ for $2 \mathrm{~T}$ using $\Delta \mathrm{T}_{\text {lift }}$ of $3 \mathrm{~K}$ as a function of $\eta$ and $m$; (b) optimal $\eta$ (left-y and circular symbols) and maximum $T E C_{r e v}$ (right-y and triangular symbols) for $2 \mathrm{~T}$ using $\Delta T_{\text {lift }}$ of $3 \mathrm{~K}$ (solid symbols) and $10 \mathrm{~K}$ (hollow symbols) as a function of $m$.

\section{Conclusions}

The relation between thermal hysteresis and the reversible MC performance in materials undergoing a magnetoelastic transition was discussed in the framework of the Bean-Rodbell model using Temperature-averaged Entropy Change (TEC) as a figure of merit. It is shown that the reversible TEC experiences a significant increase above a certain magnetic field change that overcomes the existing thermal hysteresis at zero field. That magnetic field was found to follow a strong dependence with the $\eta$ parameter and the atomic moment for FOPT. In addition, it was shown that FOPT materials with moderate $\eta$ parameter (around 1.3-1.8) are more suitable than SOPT materials at conventional magnetic field changes for all the atomic moments and temperature spans between 3 and $10 \mathrm{~K}$. Moreover, it was shown that larger atomic moment leads to higher reversible TEC values due to the significant influence on the magnetic field evolution of the transition temperatures.

Author Contributions: Conceptualization of the work by V.F. and L.M.M.-R.; resources by V.F.; software by L.M.M.-R.; data analysis and validation by L.M.M.-R. and V.F.; original draft preparation by L.M.M.-R.; review and editing by V.F. and L.M.M.-R. All authors have read and agreed to the published version of the manuscript.

Funding: Work supported by AEI/FEDER-UE (grant PID2019-105720RB-I00), US/JUNTA/FEDERUE (grant US-1260179), Consejería de Economía, Conocimiento, Empresas y Universidad de la Junta de Andalucía (grant P18-RT-746), and Army Research Laboratory under Cooperative Agreement Number W911NF1920212. 
Acknowledgments: L. M. Moreno-Ramírez acknowledges his postdoctoral contract from Junta de Andalucía and European Social Fund (ESF).

Conflicts of Interest: The authors declare no conflict of interest.

\section{References}

1. Brown, G.V. Magnetic heat pumping near room temperature. J. Appl. Phys. 1976, 47, 3673-3680. [CrossRef]

2. Pecharsky, V.K.; Gschneidner, K.A. Giant magnetocaloric effect in $\mathrm{Gd}_{5}\left(\mathrm{Si}_{2} \mathrm{Ge}_{2}\right)$. Phys. Rev. Lett. 1997, 78, 4494-4497. [CrossRef]

3. Franco, V.; Blázquez, J.S.; Ipus, J.J.; Law, J.Y.; Moreno-Ramírez, L.M.; Conde, A. Magnetocaloric effect: From materials research to refrigeration devices. Prog. Mater. Sci. 2018, 93, 112-232. [CrossRef]

4. Zimm, C.; Jastrab, A.; Sternberg, A.; Pecharsky, V.; Gschneidner, K.; Osborne, M.; Anderson, I. Description and Performance of a Near-Room Temperature Magnetic Refrigerator. In Advances in Cryogenic Engineering; Springer: Berlin, German, 1998; pp. 1759-1766, ISBN 978-1-4757-9047-4.

5. Kitanovski, A.; Tušek, J.; Tomc, U.; Plaznik, U.; Ožbolt, M.; Poredoš, A. Magnetocaloric Energy Conversion-From Theory to Applications; Springer: Berlin, German, 2015; ISBN 978-3-319-08741-2.

6. Tishin, A.M.; Spichkin, Y.I. The Magnetocaloric Effect and Its Applications; Institute of Physics: Bristol, UK; Philadelphia, PA, USA, 2003; ISBN 9781420033373.

7. Jaeger, G. The Ehrenfest classification of phase transitions: Introduction and evolution. Arch. Hist. Exact Sci. 1998, 53, 51-81. [CrossRef]

8. Gottschall, T.; Skokov, K.P.; Fries, M.; Taubel, A.; Radulov, I.; Scheibel, F.; Benke, D.; Riegg, S.; Gutfleisch, O. Making a Cool Choice: The Materials Library of Magnetic Refrigeration. Adv. Energy Mater. 2019, 9, 1901322. [CrossRef]

9. Franco, V.; Blázquez, J.S.; Ingale, B.; Conde, A. The magnetocaloric effect and magnetic refrigeration near room temperature: Materials and models. Annu. Rev. Mater. Res. 2012, 42, 305-342. [CrossRef]

10. Gutfleisch, O.; Gottschall, T.; Fries, M.; Benke, D.; Radulov, I.; Skokov, K.P.; Wende, H.; Gruner, M.; Acet, M.; Entel, P.; et al. Mastering hysteresis in magnetocaloric materials. Philos. Trans. R. Soc. A Math. Phys. Eng. Sci. 2016, 374, 20150308. [CrossRef]

11. Zverev, V.I.; Tishin, A.M.; Kuz'min, M.D. The maximum possible magnetocaloric $\Delta$ T effect. J. Appl. Phys. 2010, 107, 043907. [CrossRef]

12. Sandeman, K.G. Magnetocaloric materials: The search for new systems. Scr. Mater. 2012, 67, 566-571. [CrossRef]

13. Guillou, F.; Yibole, H.; Porcari, G.; Zhang, L.; Van Dijk, N.H.; Brück, E. Magnetocaloric effect, cyclability and coefficient of refrigerant performance in the $\mathrm{MnFe}(\mathrm{P}, \mathrm{Si}, \mathrm{B})$ system. J. Appl. Phys. 2014, 116, 063903. [CrossRef]

14. Qian, S.; Nasuta, D.; Rhoads, A.; Wang, Y.; Geng, Y.; Hwang, Y.; Radermacher, R.; Takeuchi, I. Not-in-kind cooling technologies: A quantitative comparison of refrigerants and system performance. Int. J. Refrig. 2016, 62, 177-192. [CrossRef]

15. Moya, X.; Defay, E.; Heine, V.; Mathur, N.D. Too cool to work. Nat. Phys. 2015, 11, 202-205. [CrossRef]

16. Smith, A.; Bahl, C.R.H.; Bjork, R.; Engelbrecht, K.; Nielsen, K.K.; Pryds, N. Materials challenges for high performance magnetocaloric refrigeration devices. Adv. Energy Mater. 2012, 2, 1288-1318. [CrossRef]

17. Brück, E.; Yibole, H.; Zhang, L. A universal metric for ferroic energy materials. Philos. Trans. R. Soc. A Math. Phys. Eng. Sci. 2016, 374, 20150303. [CrossRef]

18. Wood, M.E.; Potter, W.H. General analysis of magnetic refrigeration and its optimization using a new concept: Maximization of refrigerant capacity. Cryogenics (Guildf) 1985, 25, 667-683. [CrossRef]

19. Chaudhary, V.; Maheswar Repaka, D.V.; Chaturvedi, A.; Sridhar, I.; Ramanujan, R.V. Magnetocaloric properties and critical behavior of high relative cooling power FeNiB nanoparticles. J. Appl. Phys. 2014, 116, 163918. [CrossRef]

20. Fang, Y.; Yu, Z.; Peng, G.; Feng, T. Near room-temperature magnetocaloric effect in amorphous FeSc alloys: The effect of minor Co additions. J. Non. Cryst. Solids 2019, 505, 211-214. [CrossRef]

21. Duc, N.T.M.; Shen, H.X.; Thiabgoh, O.; Huong, N.T.; Sun, J.F.; Phan, M.H. Melt-extracted $\mathrm{Gd}_{73.5} \mathrm{Si}_{13} \mathrm{~B}_{13.5} / \mathrm{GdB}_{6}$ ferromagnetic/antiferromagnetic microwires with excellent magnetocaloric properties. J. Alloys Compd. 2020, 818, 153333. [CrossRef]

22. Griffith, L.D.; Mudryk, Y.; Slaughter, J.; Pecharsky, V.K. Material-based figure of merit for caloric materials. J. Appl. Phys. 2018, 123, 034902. [CrossRef]

23. Jacobs, S.; Auringer, J.; Boeder, A.; Chell, J.; Komorowski, L.; Leonard, J.; Russek, S.; Zimm, C. The performance of a large-scale rotary magnetic refrigerator. Int. J. Refrig. 2014, 37, 84-91. [CrossRef]

24. Lei, T.; Nielsen, K.K.; Engelbrecht, K.; Bahl, C.R.H.; Neves Bez, H.; Veje, C.T. Sensitivity study of multi-layer active magnetic regenerators using first order magnetocaloric material $\mathrm{La}(\mathrm{Fe}, \mathrm{Mn}, \mathrm{Si})_{13} \mathrm{H}_{\mathrm{y}}$. J. Appl. Phys. 2015, 118, 014903. [CrossRef]

25. Chirkova, A.; Skokov, K.P.; Schultz, L.; Baranov, N.V.; Gutfleisch, O.; Woodcock, T.G. Giant adiabatic temperature change in FeRh alloys evidenced by direct measurements under cyclic conditions. Acta Mater. 2016, 106, 15-21. [CrossRef]

26. Bean, C.P.; Rodbell, D.S. Magnetic disorder as a first-order phase transformation. Phys. Rev. 1962, 126, 104-115. [CrossRef]

27. Bez, H.N.; Nielsen, K.K.; Norby, P.; Smith, A.; Bahl, C.R.H. Magneto-elastic coupling in La(Fe, Mn, Si) ${ }_{13} \mathrm{H}_{\mathrm{y}}$ within the Bean-Rodbell model. AIP Adv. 2016, 6, 056217. [CrossRef]

28. Law, J.Y.; Franco, V.; Moreno-Ramírez, L.M.; Conde, A.; Karpenkov, D.Y.; Radulov, I.; Skokov, K.P.; Gutfleisch, O. A quantitative criterion for determining the order of magnetic phase transitions using the magnetocaloric effect. Nat. Commun. 2018, 9, 2680. [CrossRef] 
29. Karpenkov, D.Y.; Karpenkov, A.Y.; Skokov, K.P.; Radulov, I.A.; Zheleznyi, M.; Faske, T.; Gutfleisch, O. Pressure Dependence of Magnetic Properties in $\mathrm{La}(\mathrm{Fe}, \mathrm{Si})_{13}$ : Multistimulus Responsiveness of Caloric Effects by Modeling and Experiment. Phys. Rev. Appl. 2020, 13, 034014. [CrossRef]

30. Piazzi, M.; Bennati, C.; Curcio, C.; Kuepferling, M.; Basso, V. Modeling specific heat and entropy change in La(Fe-Mn-Si) $13-\mathrm{H}$ compounds. J. Magn. Magn. Mater. 2016, 400, 349-355. [CrossRef]

31. Amaral, J.S.; Das, S.; Amaral, S.V. The Mean-Field Theory in the Study of Ferromagnets and the Magnetocaloric Effect. In Thermodynamics-Systems in Equilibrium and Non-Equilibrium; IntechOpen: London, UK, 2011. [CrossRef]

32. Greenwood, N.N.; Earnshaw, A. Chemistry of the Elements; Butterworth-Heinemann: Oxford, UK, $2012 ;$ ISBN 9780080501093.

33. Kaeswurm, B.; Franco, V.; Skokov, K.P.; Gutfleisch, O. Assessment of the magnetocaloric effect in La, $\operatorname{Pr}(\mathrm{Fe}, \mathrm{Si}) \mathrm{under}$ cycling. J. Magn. Magn. Mater. 2016, 406, 259-265. [CrossRef]

34. Shen, J.; Wang, F.; Zhao, J.L.; Wu, J.F.; Gong, M.Q.; Hu, F.X.; Li, Y.X.; Sun, J.R.; Shen, B.G. Reduction in hysteresis losses and large magnetic entropy change in the B-doped $\mathrm{La}(\mathrm{Fe}, \mathrm{Si})_{13}$ compounds. J. Appl. Phys. 2010, 107, 09A909. [CrossRef]

35. Moreno-Ramírez, L.M.; Blázquez, J.S.; Radulov, I.A.; Skokov, K.P.; Gutfleisch, O.; Franco, V.; Conde, A. Combined kinetic and Bean-Rodbell approach for describing field-induced transitions in $\mathrm{LaFe}_{11.6} \mathrm{Si}_{1.4}$ alloys. J. Phys. D Appl. Phys. 2021, $54,135003$. [CrossRef]

36. Guillou, F.; Porcari, G.; Yibole, H.; Van Dijk, N.; Brück, E. Taming the first-order transition in giant magnetocaloric materials. Adv. Mater. 2014, 26, 2671-2675. [CrossRef] [PubMed]

37. Giguère, A.; Foldeaki, M.; Ravi Gopal, B.; Chahine, R.; Bose, T.K.; Frydman, A.; Barclay, J.A. Direct Measurement of the Giant Adiabatic Temperature Change in $\mathrm{Gd}_{5} \mathrm{Si}_{2} \mathrm{Ge}_{2}$. Phys. Rev. Lett. 1999, 83, 2262-2265. [CrossRef]

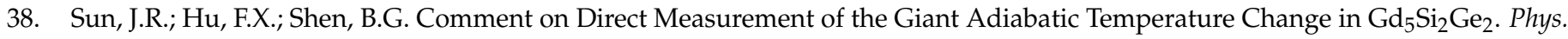
Rev. Lett. 2000, 85, 4191. [CrossRef] [PubMed]

39. Planes, A.; Mañosa, L.; Acet, M. Magnetocaloric effect and its relation to shape-memory properties in ferromagnetic Heusler alloys. J. Phys. Condens. Matter 2009, 21, 233201. [CrossRef]

40. Palacios, E.; Bartolomé, J.; Wang, G.; Burriel, R.; Skokov, K.; Taskaev, S.; Khovaylo, V. Analysis of the Magnetocaloric Effect in Heusler Alloys: Study of $\mathrm{Ni}_{50} \mathrm{CoMn}_{36} \mathrm{Sn}_{13}$ by Calorimetric Techniques. Entropy 2015, 17, 1236-1252. [CrossRef] 